Kotelnikov, V. A., et al. (1962a), Resultat radiolokatsii vener, Radiotechn. i Electron. 7, No. 11, 1860-1872.

Kotelnikov, V. A., et al. (1962b), Radar observations of the planet Mercury, Dokl. Akad. Nauk. 148, No. 6, 1320-1323.

Kotelnikov, V. A., et al. (1963a), Radar observations of Venus in the Soviet Union in 1962, Dokl. Akad. Nauk. 151, No. 3, 532-535.

Kotelnikov, V. A., et al. (1963b), Radar studies of the planet Mars in the Soviet Union, Dokl. Akad. Nauk. 151, No. 4, 811-814.

Kotelnikov, V. A., et al. (1964), Radar observations of the planet Venus, Dokl. Akad. Nauk. 155, No. 5, 1035-1038.

Maron, I., G. Luchak, and W. Blitzstein' (1961), Radar observations of Venus, Science 134, 1419-1420.

Mayer, C. H. (1961), The Solar System, Vol. 3, Planets and Satellites, ch. 12, pp. 442-472, ed. G. P. Kuiper, and B. M. Middlehurst (Univ. of Chicago Press, Chicago).

Muhleman, D. O. (1964), Radar scattering from Venus and the Moon, Astron. J. 69, No. 1, 34-41.

Pettengill, G. H., et al. (1962), A radar investigation of Venus, Astron. J. 67, 181-190.

Pettengill, G. H., and R. B. Dyce (1965), Paper presented at Spring URSI meeting, Wash., D.C.

Pettengill, G. H., and J. C. Henry (1962), Enhancement of radar reflectivity associated with the lunar crater Tycho, J. Geophys. Res. 67, No. 12, 4881-4885.
Ponsonby, R. E. B., J. H. Thomson, and K. S. Imrie (1964), Radar observations of Venus and a determination of the astronomical unit, M.N.R.A.S. 128, 1-17.

Price, R., et al. (1959), Radar echoes from Venus, Science 129, 751-753.

Price, R., and G. H. Pettengill (1961), Radar echoes from Venus and a new determination of the Solar Parallax, Planetary Space Sci. 5, 71-74.

Smith, W. B. (1963), Radar Observations of Venus, Astron. J. 68, No. 1, 15-21.

Thomson, J. H., et al. (1961), A new determination of the solar parallax by means of radar echoes from Venus, Nature 190, No. $4775,519-520$.

Victor, W. K., and R. Stevens (1961), Exploration of Venus by radar, Science 134, 46-48.

\title{
Preliminary Venus Radar Results ${ }^{1}$
}

\author{
Richard M. Goldstein \\ Communications Systems Research Section, Jet Propulsion Laboratory, California Institute of \\ Technology, Pasadena, Calif.
}

\section{Introduction}

This note presents some of the preliminary results obtained by the Jet Propulsion Laboratory during its radar observations of Venus. These experiments were performed during the months surrounding the inferior conjunction of June, 1964.

The radar parameters were as follows:

$$
\begin{aligned}
& \text { Power }=100 \mathrm{~kW} \\
& \text { Frequency }=2388 \mathrm{Mc} / \mathrm{s} \\
& \text { Antenna Gain }=108.5 \mathrm{~dB} \text { (two-way) } \\
& \text { System Temperature }=33^{\circ} \mathrm{K} .
\end{aligned}
$$

Several different types of experiments were performed, but here we shall report on only spectrum and range-spectrum analysis of the echo. Each planetary reflecting element has four attributes which may be isolated by the radar: velocity, time delay, intensity for normal, and intensity for crossed polarization. The methods used to isolate these attributes are described by Goldstein [1964]. ${ }^{2}$

\section{The Rotation of Venus}

Figure 1 presents the contours of constant time delay and of constant velocity (or Doppler frequency shift).

\footnotetext{
1 This paper presents the results of one phase of research carried out at the Jet Propulsion Laboratory, California Institute of Technology, under Contract No. NAS 7-100, sponsored by the National Aeronautics and Space Administration.

${ }_{2}$ Goldstein. R. M. (1964). Venus characteristics by earth-based radar, Astron. J. 69 No. 1, 12-18.
}

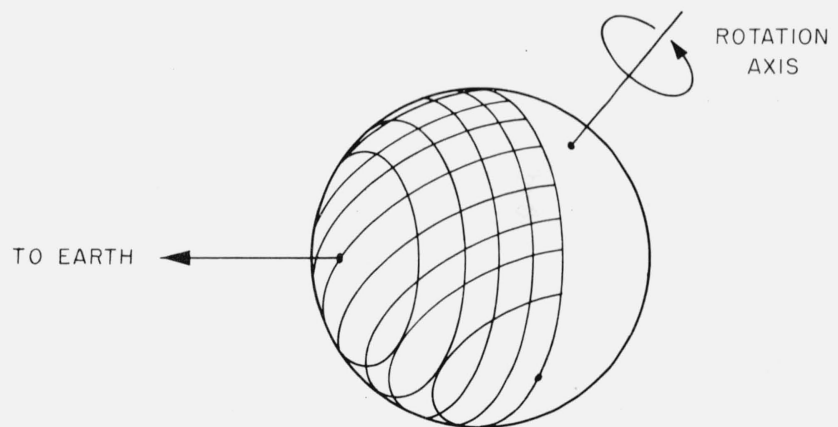

FigURE 1. Countors of constant time delay and of constant frequency shift.

The time-delay increments are $125 \mu$ sec (11 miles) and are constant. The frequency increments are not constant, but are proportional to that rotation component which is perpendicular to the line of sight. As Venus passes the Earth in its orbit, the frequency increments first decrease and then increase. This phenomonon enables the three components of Venus's rotation vector to be determined. Goldstein [1964] gives the details of this computation.

A sample of the basic data obtained is given in figure 2. The echo arising from each ring of constant time delay has first been separated by the radar and then spread into its frequency components in order 
to produce the curves of figure 2. Many things about Venus can be deduced from data such as these. The time of flight can be measured to within 11 miles by noting the first zone which contains an echo. Precision down to 2 or 3 miles may be obtained by making use of the bandwidths of the first few spectrograms. In addition, the scattering law (i.e., the dependence of the echo power on the angle of incidence) may be determined from the areas under the successive curves. Moreover, structure on spectra, such as those of figure 2, may be related to topographic features on the surface of Venus. All of these will be reported on subsequently, after data reduction has been completed.

For the perpendicular component of rotation, the bandwidths of the spectra are extrapolated to the limb-to-limb bandwidth. The results of 48 such bandwidths are plotted in figure 3 . The solid curve shows the result of adjusting the three components of Venus's spin vector for a best fit to the data. The corresponding period of rotation is $250 \pm 9$ days, retrograde. The corresponding direction of the spin axis is $-62 \pm 4^{\circ}$ declination and $81 \pm 6^{\circ}$ right ascension.

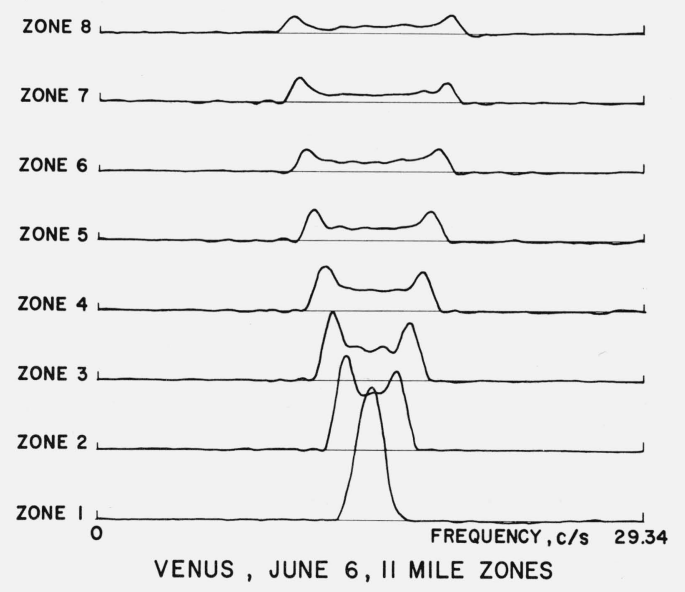

FIGURE 2. Range-gated Venus spectrograms.

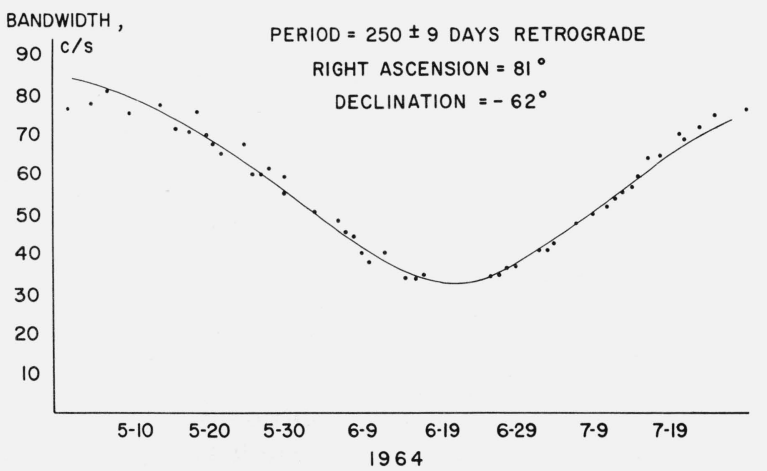

Figure 3. Plot of limb-to-limb bandwidths.

\section{Features on Venus}

Normally, circularly polarized waves are transmitted and the opposite sense of polarization is received. Since a smooth reflector reverses the polarization, the normal mode produces the strongest echo. However, multiple reflections can be expected to occur in regions where the surface is rough compared to the wave length.

In a search for these areas, the same sense of polarization was both transmitted and received. Figure 4 shows several such spectrograms, taken on several different days.

Normal spectrograms of the Venus echo show a large, sharp central spike (see Goldstein, [1964]). However, the polarization technique completely suppresses this spike and allows topographic features to be seen clearly. Two such features produced the peaks shown in figure 4. The peaks were observed to move slowly across the spectrograms, from the high-frequency side to the low, carried by Venus's rotation.

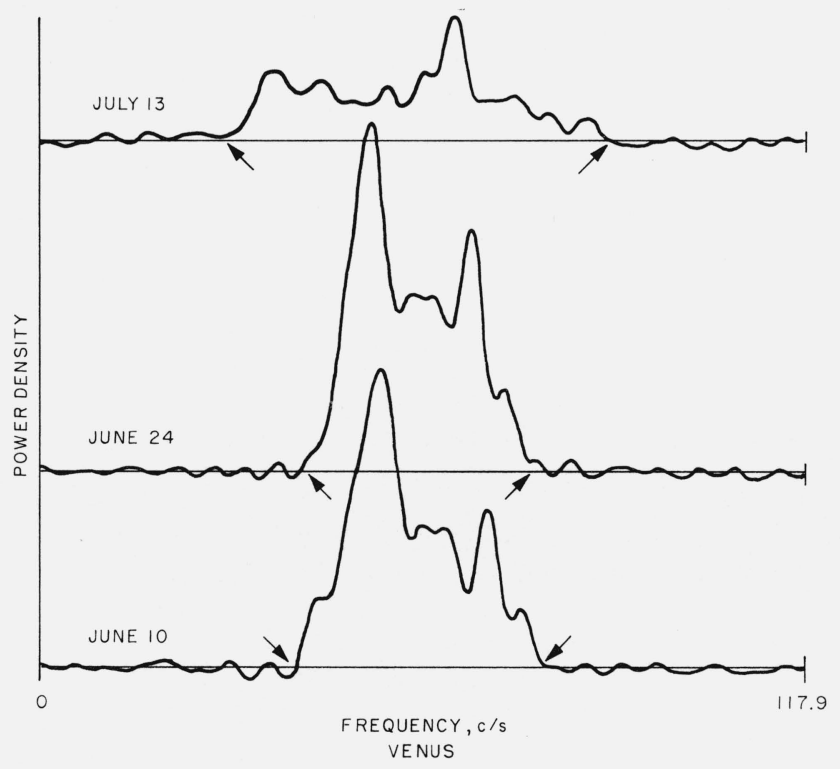

FIGURE 4. Venus spectrograms taken with reversed polarization.

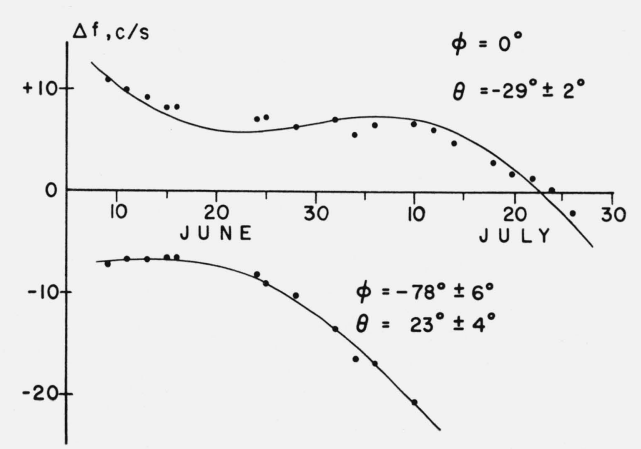

Figure 5. Plot of frequency deviation of spectral peaks. 
By observing the motions of these peaks across the spectrograms, one may compute the locations of the topographic features which produced them. From each depolarized spectrum, the frequency deviation of each peak has been plotted. A reproduction of this plot is given in figure 5 . The solid curves represent locations on Venus which produce the best fit to the data, and the corresponding Venus-centered longitudes and latitudes are marked in the figure. The Venus rotation vector as described above was used in this computation. The fact that the theoretical curves fit the data so well indicates that the reflecting objects are, indeed, fixed to the planetary surface, and that an accurate rotation vector has been used.

Figure 6 describes the Venus-centered coordinate system. The North direction was chosen along Venus's rotation axis, but opposite to the usual direction since the rotation is retrograde. Thus, North is toward the celestial north pole. The prime meridian was chosen to go through the sharpest of the two features in the spectrograms. This meridiah contained the sub-Earth point on 23 July 1964. The positive direction in longitude is such that the sub-Earth point moves through successively larger angles. Figure 6 gives the locations of the "centers of reflection" of the two Venusian features, but the objects may be much larger than shown. Although the technique used gives a good indication of the longitudinal extent of the features, it gives little indication of their latitudinal extent.

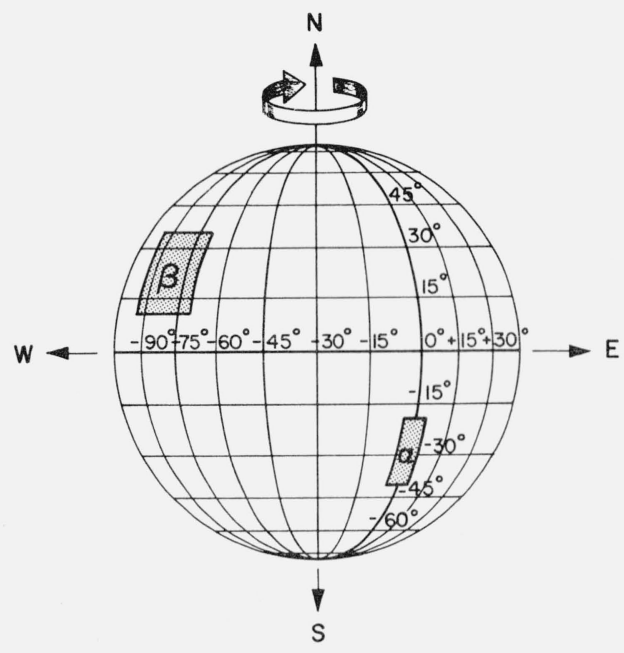

Figure 6. Venus-centered coordinate sysiem.

When the range-spectrum data has been reduced, additional features will be located in the equatorial belt of Venus.

\title{
Preliminary Mars Radar Results
}

\author{
Richard M. Goldstein
}

\section{Communications Systems Research Section, Jet Propulsion Laboratory, California Institute of Technology, Pasadena, Calif.}

This note presents some very preliminary results obtained by the Jet Propulsion Laboratory during its recent radar observations of Mars. These experiments were performed nearly every night of February, March, and half of April 1965. The radar parameters were the same as described in the previous paper in this same issue of Venus results, except the system temperature was lowered to $27^{\circ} \mathrm{K}$. However, since the power returned by Mars is less than Venus by a factor of almost 100, and Doppler broadening is greater by a factor of over 200, only spectral data was taken. That is, pure CW was transmitted for the $11 \mathrm{~min}$ time-of-flight, and then the echo was received for $11 \mathrm{~min}$. The received signal was then analyzed into its frequency spectrum.

Altogether, almost 1300 such runs were obtained, each covering a bandwidth of $3700 \mathrm{c} / \mathrm{s}$ with a frequency resolution of $84 \mathrm{c} / \mathrm{s}$. We note that the limb-to-limb Doppler broadening which Mars produces is $7670 \mathrm{c} / \mathrm{s}$. Thus, the small amount of power which is returned outside of the $3700-\mathrm{c} / \mathrm{s}$ bandwidth is not detected.

Although the power received in each run originates from most of the planetary disk, the largest portion of the power is returned from the sub-Earth point. Consequently, the runs were averaged according to the longitude of the corresponding sub-Earth points. The result is a set of 36 spectrograms, each the average of over 33 runs, which corresponds to $10^{\circ}$ increments of Martian longitude. The latitude of the sub-Earth point was close to $+21^{\circ}$ during the entire experiment.

${ }^{1}$ This paper presents the results of one phase of research carried out at the Jet Propulsion Laboratory, California Institute of Technology, under Contract No. NAS 7-100, sponsored by the National Aeronautics and Space Administration. 


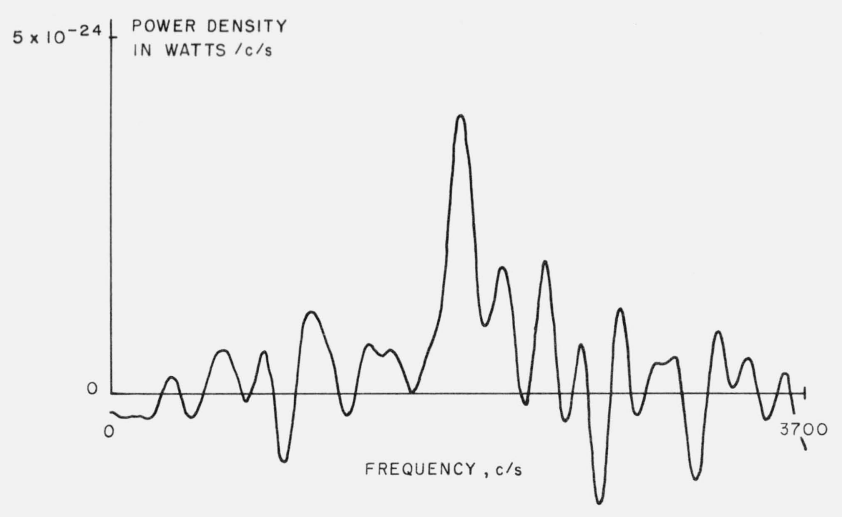

Figure 1. Mars spectrogram, $180^{\circ}$ to $190^{\circ}$ longitude.

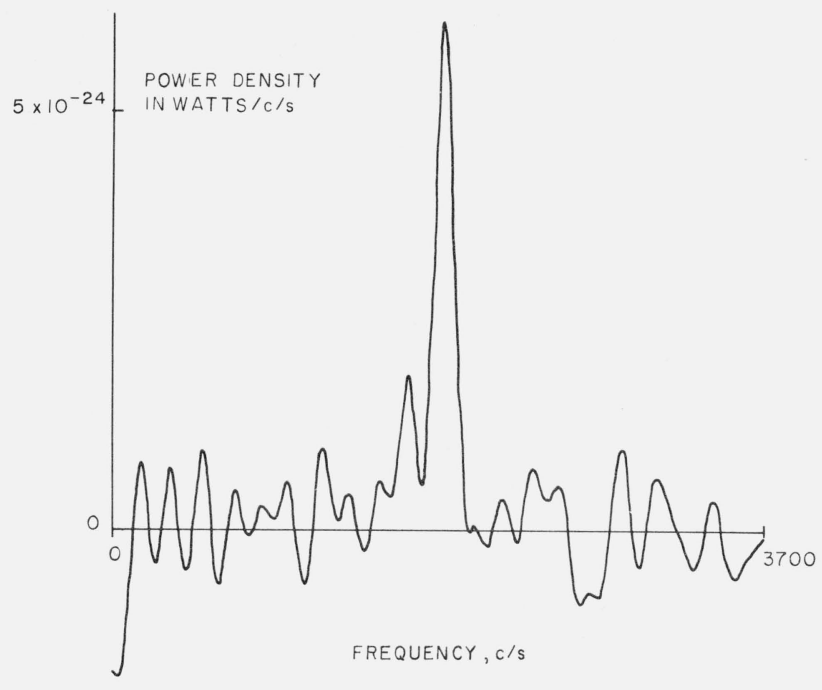

Figure 3. Mars spectrogram, $200^{\circ}$ to $210^{\circ}$ longitude.

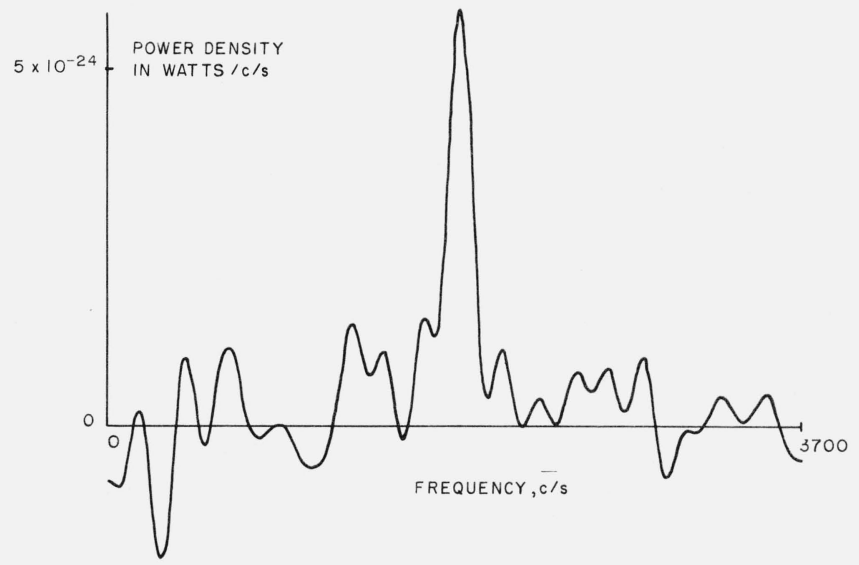

FigURE 2. Mars spectrogram, $190^{\circ}$ to $200^{\circ}$ longitude.

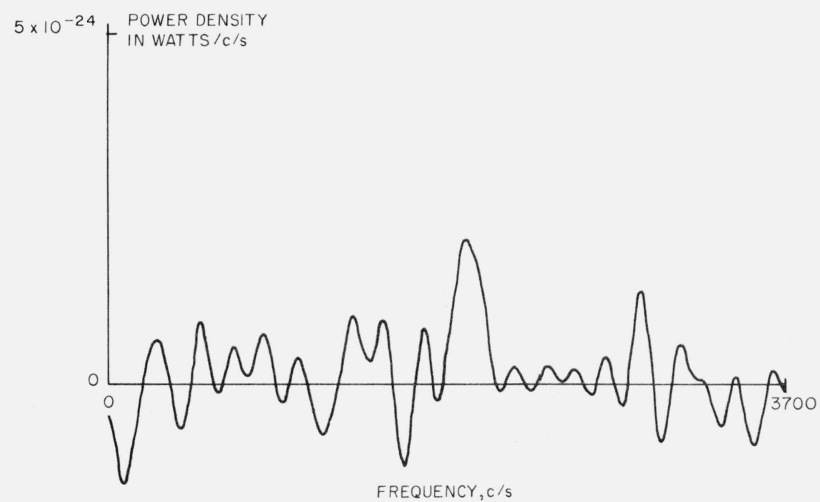

Figure 4. Mars $\begin{gathered}\text { spectrogram, } 210^{\circ} \text { to } 220^{\circ} \\ \text { longitude. }\end{gathered}$

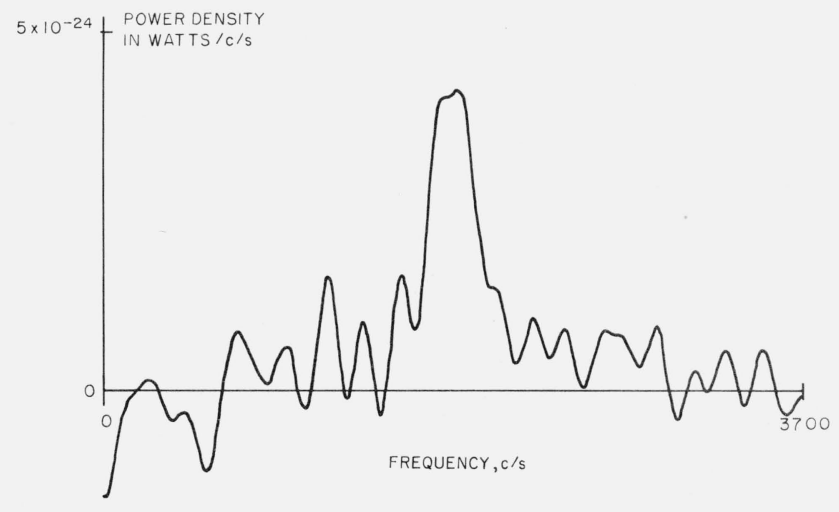

Figure 5. Mars spectrogram, $240^{\circ}$ to $250^{\circ}$ longitude. 
Most of these spectrograms show a small amount of power reflected diffusely by the disk. Some of them, however, show relatively strong, narrowband reflections, which originate from an area less than $2^{\circ}$ in extent about the sub-Earth point.

Figures 1 through 4 are samples of these spectrograms from successive $10^{\circ}$ strips of Mars. The narrowband echo is seen to increase, reaching a maximum at $200^{\circ}$ to $210^{\circ}$ (the region of Trivium Charontis), and then it drops off rather abruptly. One may conclude that there is a very smooth region on Mars, extending $20^{\circ}$ to $30^{\circ}$ in longitude, and of unknown latitudinal extent.

A similar, but wider band, sequence of echoes was reflected from the $240^{\circ}$ to $250^{\circ}$ region. Figure 5 is a sample spectrogram.

When data reduction is complete, both returned power and bandwidth will be presented as functions of longitude, and compared to the optical features on Mars. It may also be possible to show some of the regions of stronger reflectivity as functions of time as well.

(Paper 69D12-611)

\title{
Recent Ârecibo Observations of Mercury
}

\author{
G. H. Pettengill
}

Arecibo Ionospheric Observatory, ${ }_{1}^{1}$ Arecibo, Puerto Rico

As the new determination of Mercury's rotation is at variance with four generations of optical work and is not confirmed by JPL workers, it is appropriate to further discuss the Arecibo procedures.

Mercury moved into the Arecibo beam on 11 March 1965 with strongest echoes predicted for 7 April 1965. Although this conjunction should have given echo strengths slightly inferior to the conjunction of May 1964, improvements in receiver noise figure and data handling technique have more than made up for the less favorable geometry.

${ }^{1}$ Operated by Cornell University with the support of the Advanced Research Projects Agency under a research contract with the Air Force Office of Scientifi Research, OAR.

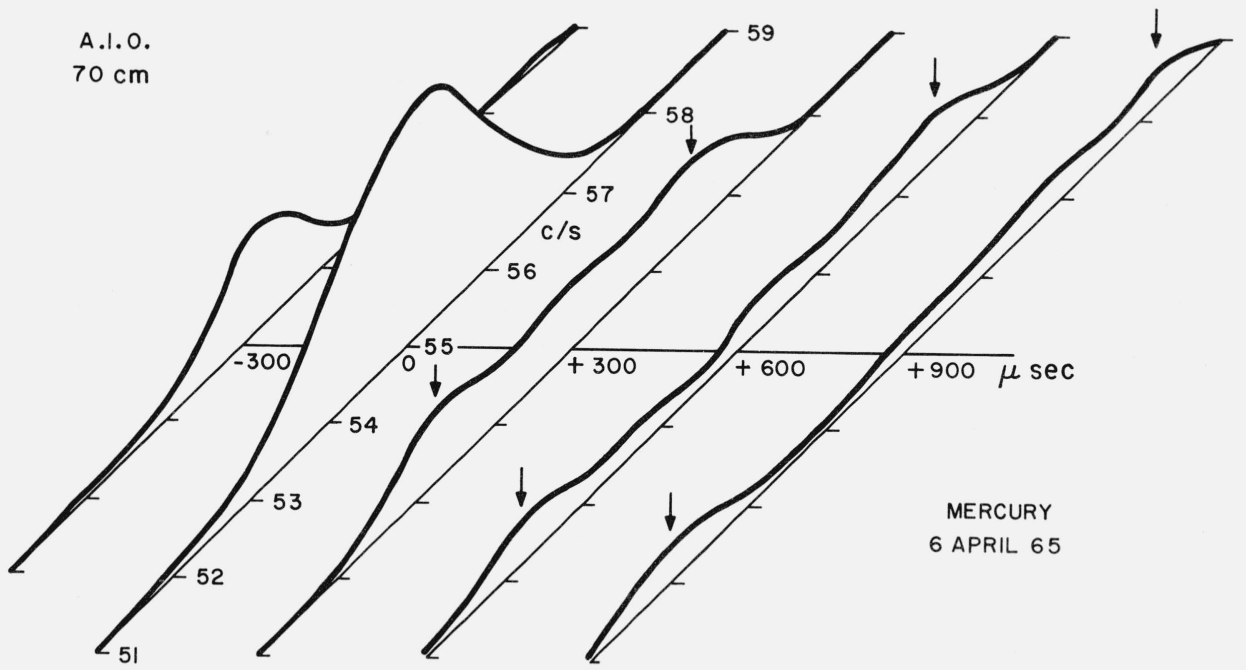

FIGURE 1. Plot of echo power as a function of relative delay (toward the right) and Doppler frequency shift (diagonally).

The arrows indicate the "wings", whose separation, when combined with the distance from the echo maximum, gives the instan taneous apparent rotation rate. 